\title{
12 Parametrische und nichtparametrische Methoden der Einzelfallstatistik
}

von C. Möbus, G. Göricke und P. Kröh

\section{1 EINLEITUNG}

Die Auswertung von zeitbezogenen Daten macht i.allg. die Anwendung spezieller statistischer Verfahren notwendig. Die Begründung hierfür liegt in der sog. "Abhängigkeit" der Daten: Man nimmt im Gegensatz zur Querschnittuntersuchung an, daß die Daten eine gewisse "Trägheit" oder ein "Beharrungsvermögen" aufweisen. Diesen Effekt kann man auf physiologische Prozesse oder psychologische Ursachen wie "Erinnerungs"- und "Gewöhnungseffekte" oder auch regelmäßig wiederkehrende äußere Einflüsse (wie z.B. regelmäßige Berufstätigkeit) zuruickführen. Diese zeitliche Abhängigkeit der Daten wird a uch Autokorreliertheit genannt. Die Verwendung "normaler" statistischer Verfahren zur Analyse autokorrelierter Daten liefert verzerrte Prüfgrößen, die zu falschen Interpretationen führen.

Abhilfe schaffen die von uns vorgestellten Methoden. Zur besonderen Veranschaulichung haben wir sie mit einem aus der Literatur bekannten Beispiel durchgerechnet. Dabei erweisen sich die verteilungsfreien Randomisierungstests für den Praktiker wegen des geringeren Aufwands als geeigneter. Die verteilungsgebundenen Verfahren setzen in jedem Fall den Zugang zu einem Computer voraus.

12.2 VERTEILUNGSFREIE VERFAHREN: RANDOMISIERUNGS- BZW. PERMUTATIONSTESTS

Einfache Auswertungsverfahren, die speziell für Zeitreihenexperimente geeignet sind, bieten sich mit dem Randomisierungs-oder Permutationstest an (Edgington 1967, 1969a, b, 1971, 1973,1975a, b, 1980). Dabei geht die prinzipielle Konstruktionsidee auf Fisher (1951) zurück. Hährend die Prüfgrößen z.T. völlig äquivalent zu denen der $k$ lassischen statistischen Verfahren ( $t$ - oder F-Bruch, aber auch einfache Mittelwert-oder Mediandifferenzen) sein können, weichen Nullhypothesenformulierung und Inferenzmodell doch wesentlich vom klassischen Verfahren ab.

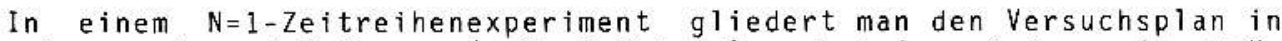
mehrere $A-$ und $B-P h a s e n\left(z . B . A_{1} B_{1} A_{2} B_{2}\right)$, wobei $A_{1}$ meistens eine präexperimentelle Beobachtungsphase darstelit. Diese wird dann von einer Treatment- oder B-Phase abgelöst. Dann folgt wieder eine reine Beobachtungs- oder Löschungsphase $A_{2}$ usw. Die Nullhypothese $H_{0}$ ("Kein Unterschied $z$ wischen A- und B-Phasen") wird geprüft im Vergleich mit einer bedingten Wahrscheinlichkeitsverteilung, die sich aus allen theoretisch möglichen Aufteilungen dieser gerade erhobenen Daten auf die Treatments A und B ergibt. Unter der Gijtigkeit der Nullhypothese liegen keine Treatmenteffekte vor. Die Daten spiegeln nur Zufallsein- 
flusse wider. Damit sind die Aufteilungen der Daten gerechtfertigt. Die so bestimmten Mittelwertunterschiede weisen ebenfalls nur Zufallseinflüsse auf. Wir erhalten unter $H_{0}$ eine Verteilung mit Mittelwertunterschieden. Diese Verteilung spielt die Rolle einer "Prüfverteilung", an der wir die empirisch vorgefundene Mittelwertdifferenz $\bar{Y}_{B}-\bar{Y}_{A}$ prüfen. Statt des Mittelwertes können auch andere Zentralitätsmaße (wie Mediane, Proportionen etc.) Verwendung finden.

Die Reduktion der Daten zu Phasenmittelwerten (oder anderen Summationsmaßen) bringt, wie Levin et aT. (1978) gezeigt haben, beträchtliche Vorteile bei der Anlayse autokorrelierter Daten. Die Mittelwerte weisen eine stark reduzierte Autokorrelation gegenuber der originalzeitreihe auf.

Die Nullhypothese $H_{0}$ wird verworfen, wenn es unwahrscheinlich ist $(p<0,05)$, daß man durch einfache Umbenennungen der Phasen eine gleichgroße oder größere Mittelwertdifferenz als die beobachtete erhält. Howird beibehalten, wenn einfache Umbenennungen der experimentellen "Phasen gleiche oder größere Unterschiede als die beobachteten häufig auftreten lassen. Somit beschäftigt sich Inferenz nicht mehr, wie bei kTassischen Experimenten, mit der Generalisation iber Personen und Situationen, sondern mit der Generalisation über andere Avs. B-Zuweisungen. Lehman (1975) bezeichnete dieses Inferenzmodell als "Randomisierungsmodel1", das sich logisch vom ublichen "Stichproben-Populations-Model " abhebt. Es werden keine Populationsannahmen gemacht, da die beobachteten Daten die Rolle der Population ibernehmen.

Zur Illustration betrachten wir das folgende Beispiel (vgl. Möbus u. Nagl 1983) für die Auswertung des häufig verwendeten $A_{1} B_{1} A_{2} B_{2}-D e-$ signs. Im Experiment wurden die in Tabelle 1 dargesteliten Daten beobachtet.

Tabelle 1. Daten aus einem Experiment, dem ein $A_{1} B_{1} A_{2} B_{2}-$ Design zugrunde liegt. (Aus Möbus u. Nag1, 1983, S.289; Nachdruck mit Genehmigung des Verlags J.C. Hogrefe)

\begin{tabular}{|c|c|c|c|c|}
\hline 1. & $\begin{array}{l}2 . \\
B_{1}\end{array}$ & $\begin{array}{l}3 . \\
\mathrm{A}_{2}\end{array}$ & $\begin{array}{l}\text { 4. Phase } \\
B_{2}\end{array}$ & \\
\hline 2.0 & $\begin{array}{r}7.0 \\
(7.5-\end{array}$ & $=3.0$ & $\frac{8.0}{\left.\bar{Y}_{\Lambda}\right)}=5.0$ & $\begin{array}{l}\text { Mittelwerte } \bar{Y}_{\tau} \\
\text { ODifferenz }\end{array}$ \\
\hline
\end{tabular}

Da das Experiment $n_{A}=2$ A-Phasen und $n_{B}=2 B$-Phasen, d. $h$. insgesamt $n_{A}+n_{B}=4$ Phasen umfábt, 1assen sich insgesamt $\left(n_{A}+n_{B}\right) ! /\left(n_{A} ! \cdot n_{B} !\right)=$ $=4 ! /(2 ! \cdot 2 !)=6$ Anordnungen (Permutationen) der $A-$ und $B-P h a s e n$ finden (Tabelle 2).

Da die Wahrscheinlichkeit, durch eine andere Aufteilung eine mindestens ebenso große Mittelwertdifferenz wie die beobachtete zu finden, größer als 0,05 ist (nämlich exakt 1/6), wird die Nullhypothese beibehalten. Da diese $P=1 / 6=0,16 \mathrm{nach}$ den üblichen Standards zu groß ist, kann das $A_{1} B_{1} A_{2} B_{2}$-Design nicht uber Mittelwertvergleiche innerhalb des Randomisierungsinferenzmodells ausgewertet werden! 
Tabelle 2. Mögliche Anordnungen der $A$ - und $B-P h a s e n$ des $A_{1} B_{1} A_{2} B_{2}-$ Designs. (Aus Möbus u. Nagl, 1983, S.290; Nachdruck mit Genehmigung des Verlags J.C.Hogrefe)

\begin{tabular}{cccccccc}
\hline Aufteilung & 1. & 2. & 3. & 4. Phase & $\overline{\mathrm{Y}}_{\mathrm{A}^{\prime}}$ & $\overline{\mathrm{Y}}_{\mathrm{B}^{\prime}}$ & $\overline{\mathrm{Y}}_{\mathrm{B}^{\prime}}-\overline{\mathrm{Y}}_{\mathrm{A}^{\prime}}$ \\
\hline 1 & $\mathrm{~A}_{1}^{\prime}$ & $\mathrm{A}_{2}^{\prime}$ & $\mathrm{B}_{1}^{\prime}$ & $\mathrm{B}_{2}^{\prime}$ & 4.5 & 5.5 & 1.0 \\
2 & $\mathrm{~A}_{1}^{\prime}$ & $\mathrm{B}_{1}^{\prime}$ & $\mathrm{A}_{2}^{\prime}$ & $\mathrm{B}_{2}^{\prime}$ & 2.5 & 7.5 & 5.0 \\
3 & $\mathrm{~A}_{1}^{\prime}$ & $\mathrm{B}_{1}^{\prime}$ & $\mathrm{B}_{2}^{\prime}$ & $\mathrm{A}_{2}^{\prime}$ & 5.0 & 5.0 & 0.0 \\
4 & $\mathrm{~B}_{1}^{\prime}$ & $\mathrm{A}_{1}^{\prime}$ & $\mathrm{A}_{2}^{\prime}$ & $\mathrm{B}_{2}^{\prime}$ & 5.0 & 5.0 & 0.0 \\
5 & $\mathrm{~B}_{1}^{\prime}$ & $\mathrm{A}_{1}^{\prime}$ & $\mathrm{B}_{2}^{\prime}$ & $\mathrm{A}_{2}^{\prime}$ & 7.5 & 2.5 & -5.0 \\
6 & $\mathrm{~B}_{1}^{\prime}$ & $\mathrm{B}_{2 .}^{\prime}$ & $\mathrm{A}_{1}^{\prime}$ & $\mathrm{A}_{2}^{\prime}$ & 5.5 & 4.5 & -1.0 \\
\hline
\end{tabular}

Um ein kleineres $p z u$ erhalten, müssen gewichtete Alternativhypothesen, wie $z . B . \bar{Y}_{A 1}<\bar{Y}_{A 2}<\bar{Y}_{B 1}<\bar{Y}_{B 2}$, geprüft werden. Hieraus leitet sich das ordinale Gewichtungsschema ab: Die $\bar{Y}_{t}$ werden entsprechend der prognostizierten Größe mit ihren Rängen gewichtet: $g_{A 1}=1, g_{A 2}=2$, $g_{B 1}=3, g_{B 2}=4$. Mit diesen Gewichten werden die empirisch erhaltenen Maße (Bittelwerte, Mediane, Proportionen) multipliziert und aufsummiert. Damit ergibt sich für das obige Beispiel:

$$
\Gamma_{\text {emp }}=\sum_{t=1}^{4} g_{1} \bar{y}_{t}=1 \cdot 2,0+2 \cdot 3,0+3 \cdot 7,0+4 \cdot 8,0=61,0 .
$$

Es gibt insgesamt $4 !=24$ Möglichkeiten, für 4 Phasen eine gewichtete Alternativhypothese aufzustellen. Die Verteilung dieser gewichteten Summen $\Gamma_{k}$ ist in Tabelle 3 dargestellt.

Da die beobachtete Reihe von Mittelwerten in einer ordinal gewichteten Summe $\Gamma$ von 61 resultiert, deren Auftretenswahrschein 7 ichkeit unter der Nullhypothese $1 / 24=0,042$ ist (s. auch rechte Spalte von Tabelle 3), wird Ho zugunsten von $H_{1}$ verworfen: Die Phasen unterscheiden sich signifikant.

Levin et al. (1978) haben gezeigt, daß die durch ein ordinales Gewichtungsschema bestimmte Summe $\Gamma_{k}$ annähernd normalverteilt ist, wenn der Versuchsplan mindestens $4 \mathrm{~A}$ - und $4 \mathrm{~B}$-Phasen umfaßt. Somit läßt sich remp mit Hilfe der folgenden Prüstatistik auf Signifikanz überprüfen:

$$
z_{\text {emp }}=\frac{\Gamma_{\text {emp }}-E(\Gamma)}{\sqrt{\operatorname{Var}(\Gamma)}}
$$

mit

$$
\left.E(\Gamma)=\frac{1}{n} \cdot\left\{\left(\sum_{t=1}^{n} g_{t}\right) \stackrel{n}{\sum_{t=1}^{n}} \bar{y}_{t}\right)\right\}
$$




$$
\operatorname{Var}(r) \cong \frac{1}{n-1} \cdot\left\{\sum_{t=1}^{n}\left(g_{t}-\bar{g}\right)^{2}\right\} \cdot\left\{\sum_{t=1}^{n}\left(\bar{y}_{t}-\bar{y}\right)^{2}\right\} .
$$

Statt eines ordinalen Gewichtungsschemas ist auch ein Intervall-oder Verhältnisschema (nichtordinale Gewichtungsschemata) denkbar (vgl. Möbus u. Nagl 1983 , S. $291 \mathrm{ff}$.).

Tabelle 3. Verteilung der gewichteten Summen $\Gamma_{k}$ unter der Nullhypothese in einem $A_{1} B_{1} A_{2} B_{2}$-Design. (Aus Möbus ü. Nag1, 1983, S.291; Nachdruck mit Genehmigung des Verlags J.C. Hogrefe)

\begin{tabular}{|c|c|c|c|c|c|}
\hline Permutation & 1. & 2. & 3. & 4. Experimentelle Phase & $\Gamma$ \\
\hline $\mathrm{K}$ & 2.0 & 7.0 & 3.0 & B.0 Mittelwerte & \\
\hline 1 & $\mathbf{A}_{1}^{\prime}$ & $\mathrm{A}_{2}^{\prime}$ & $\mathbf{B}_{i}^{\prime}$ & $\mathrm{B}_{2}^{\prime}$ & 57 \\
\hline 2 & $\mathbf{A}_{1}^{\prime}$ & $\mathrm{A}_{2}^{\prime}$ & $\mathrm{B}_{2}^{\prime}$ & $\mathbf{B}_{i}^{\prime}$ & 52 \\
\hline 3 & $\mathbf{A}_{\mathbf{1}}^{\prime}$ & $\mathbf{B}_{i}^{i}$ & $\mathbf{A}_{2}^{\prime}$ & $\mathbf{B}_{2}^{\prime}$ & 61 \\
\hline 4 & $\mathrm{~A}_{1}^{\prime}$ & $\mathbf{B}_{i}^{i}$ & $\mathrm{~B}_{2}^{\prime}$ & $\mathrm{A}_{2}^{\prime}$ & 51 \\
\hline 5 & $\mathrm{~A}_{1}^{\prime}$ & $\mathrm{B}_{2}^{\prime}$ & $\mathrm{A}_{2}^{\prime}$ & $\mathrm{B}_{\mathrm{i}}^{\prime}$ & 60 \\
\hline 6 & $\mathbf{A}_{1}^{\prime}$ & $\mathrm{B}_{2}^{\prime}$ & $\mathbf{B}_{i}^{\prime}$ & $\mathbf{A}_{2}^{\prime}$ & 55 \\
\hline 7 & $\mathrm{~A}_{2}^{\prime}$ & $\mathrm{A}_{\mathrm{i}}$ & $\mathbf{B}_{i}^{i}$ & $\mathrm{~B}_{2}^{\prime}$ & 52 \\
\hline 8 & $\mathrm{~A}_{2}^{\prime}$ & $\mathrm{A}_{i}$ & $B_{2}^{\prime}$ & $\mathrm{B}_{i}^{i}$ & 47 \\
\hline 9 & $\mathrm{~A}_{2}^{\prime}$ & $\mathbf{B}_{1}^{\prime}$ & $\mathrm{A}_{1}^{\prime}$ & $\mathrm{B}_{2}^{\prime}$ & 60 \\
\hline 10 & $\mathrm{~A}_{2}^{\prime}$ & $\mathbf{B}_{1}^{\prime}$ & $\mathbf{B}_{2}^{\prime}$ & $A_{1}^{\prime}$ & 45 \\
\hline 11 & $\mathrm{~A}_{2}^{\prime}$ & $\mathrm{B}_{2}^{\prime}$ & $\mathrm{A}_{1}^{\prime}$ & $B_{i}^{i}$ & 59 \\
\hline 12 & $\mathrm{~A}_{2}^{\prime}$ & $\mathrm{B}_{2}^{\prime}$ & $\mathbf{B}_{1}^{i}$ & $A_{i}^{\prime}$ & 49 \\
\hline 13 & $\mathrm{~B}_{1}^{\prime}$ & $A_{i}^{i}$ & $\mathrm{~A}_{2}^{\prime}$ & $\mathrm{B}_{2}^{\prime}$ & 51 \\
\hline 14 & $\mathrm{Bi}_{i}^{\prime}$ & $\mathrm{A}_{1}^{\prime}$ & $\mathrm{B}_{2}^{\prime}$ & $A_{2}^{\prime}$ & 41 \\
\hline 15 & $\mathrm{~B}_{;}^{\prime}$ & $\mathrm{A}_{2}^{\prime}$ & $\mathrm{A}_{1}^{\prime}$ & $\mathrm{B}_{2}^{\prime}$ & 55 \\
\hline 16 & $\mathrm{Bi}_{i}$ & $\mathrm{~A}_{2}^{\prime}$ & $\mathrm{B}_{2}^{\prime}$ & $\mathrm{A}_{\mathrm{i}}^{\prime}$ & 40 \\
\hline 17 & $\mathbf{B}_{1}^{\prime}$ & $\mathbf{B}_{2}^{\prime}$ & $\mathrm{A}_{\mathrm{i}}^{\prime}$ & $\mathrm{A}_{2}^{\prime}$ & 53 \\
\hline 18 & $\mathrm{~B}_{1}^{\prime}$ & $\mathrm{B}_{2}^{\prime}$ & $\mathrm{A}_{2}^{\prime}$ & $A_{i}^{i}$ & 48 \\
\hline 19 & $\mathrm{~B}_{2}^{\prime}$ & $\dot{A_{1}^{\prime}}$ & $\mathrm{A}_{2}^{\prime}$ & $B_{1}^{\prime}$ & 45 \\
\hline 20 & $\mathrm{~B}_{2}^{\prime}$ & $\mathrm{A}_{1}^{\prime}$ & $B_{1}^{\prime}$ & $\mathrm{A}_{2}^{\prime}$ & 40 \\
\hline 21 & $\mathrm{~B}_{2}^{\prime}$ & $A_{2}^{\prime}$ & $\mathrm{A}_{1}^{\prime}$ & $\mathbf{B}_{i}^{i}$ & 49 \\
\hline 22 & $\mathrm{~B}_{2}^{i}$ & $\mathrm{~A}_{2}^{\prime}$ & $B_{i}^{\prime}$ & $\mathrm{A}_{\mathrm{i}}^{\prime}$ & 39 \\
\hline 23 & $\mathrm{~B}_{2}^{\prime}$ & $\mathbf{B}_{\mathrm{i}}^{\prime}$ & $\mathbf{A}_{1}^{\prime}$ & $\mathrm{A}_{2}^{\prime}$ & 48 \\
\hline 24 & $B_{2}^{\prime}$ & $\mathbf{B}_{1}^{i}$ & $A_{2}^{\prime}$ & $A_{i}$ & 43 \\
\hline
\end{tabular}

12.3 VERTEILUNGSGEBUNDENE VERFAHREN: DAS TRANSFERMODELL VON BOX \& TI AO

Bei der Oberprüfung eines Interventionseffektes in Zeitreihen mit Hilfe klassischer statistischer Verfahren besteht ein grundsätzliches Hindernis in der Abhängigkeit der Daten bzw. der Residuen, so daß sich $z$.B. eine varianzanalytische Auswertung verbietet. Ein Verfahren, das die Abhängigkeit der seriell anfallenden Beobachtungen berücksichtigt, ist das Transfermodell von Box und Tiao. Hierbei werden die Zeitreihendaten in einen Interventionseffekt und einen nichtkontrollierbaren Effekt $\mathrm{N}_{\mathrm{t}}$ zerlegt $\langle\mathrm{s}$, auch $\mathrm{Gl}$. (16) $\rangle$. Während der In- 
terventionseffekt deterministisch ist, bildet $\mathrm{N}_{t}$ wiederum eine Zeitrethe, die seriell abhängig ist. Der nichtkontrollierbare ProzeB $N_{t}$ wird nach dem Vorschlag von Box und Jenkins (1976) mit einem ARIMAProzeß so dargestelit, daß die Fehler a des ARIMA-Prozesses jetzt im Gegensatz $z u$ den $N_{t}$ voneinander unabhängig sind. Erst die unabhängigen at lassen den Einsatz hypothesenprïfender Verfahren $z u$.

\subsubsection{Die Modellierung des Fehlerprozesses $\mathrm{N}_{t}$}

Die ARIMA-Modelle setzen sich aus a) dem autoregressiven ProzeB (ARProzeB), b) dem integrierenden ProzeB (I-ProzeB) und $c$ ) dem Moving

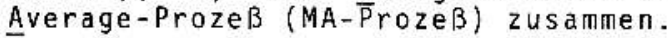

Der ARIMA(p,d,q)-Prozeß läßt sich in Rohwerten $Y_{t}$ (Hibbs 1977, S. 140) darstellen als

$$
\left(1-\phi_{1} B-\ldots-\phi_{p} B^{p}\right)(1-B)^{d} Y_{t}=\theta_{0}+\left(1-\theta_{1} B-\ldots-\theta_{q}{ }^{B}{ }^{q}\right) a_{t}
$$

oder

$$
\phi(B)(1-B)^{d} y_{t}=\theta_{0}+\theta(B) a_{t}
$$

bzw.

$$
Y_{t}=\frac{\theta_{0}+\theta(B) a_{t}}{\phi(B)(1-B)^{d}},
$$

wobei $B$ der Backshiftoperator ist: $B{ }^{k_{Y}}{ }_{t}=Y_{t-k}$. Alternative Formulierungen für das allgemeine ARIMA-Modell findet man u.a. bei Box $u$. Jenkins (1976), Glass et al. (1975), Jenkins (1979) und Gottman (1981). Der Zufallsprozeß a in Gl. (3) ist so definiert, daß er unabhängig und normalverteilt ist mit

$$
E\left(a_{t}\right)=0 \text { und } \quad \operatorname{Var}\left(a_{t}\right)=\sigma_{a}^{2} \text {. }
$$

Ein solcher Zufallsprozeß wird von einigen Autoren mit "white noise" (weißem Rauschen) und von anderen als Innovationsprozeß bezeichnet.

In G1. (3) ist $\phi(B)=1-\phi_{1} B-\ldots-\phi_{p}{ }^{P}$ ein stationärer AR-0perator der 0rdnung $p$ und $\theta(B)=1-\theta_{1} B-\ldots-\theta_{q}{ }^{q}$ ein invertierbarer MA-0perator der Ordnung $q$. Eine notwendige und hinreichende Bedingung für die Stationarität des AR-Operators bzw. der Invertierbarkeit des MA-Operators ist, daß alle Lösungen der charakteristischen Gleichung 


$$
\phi(\lambda)=1-\phi_{1} \lambda-\ldots-\phi_{p} \lambda^{p}=0
$$

bzw.

$$
\theta(\lambda)=1-\theta_{1} \lambda-\ldots-\theta_{q} \lambda^{q}=0
$$

in threm Absolutbetrag größer als eins sind. Nur wenn die Stationaritätsbedingung (Invertierbarkeitsbedingung) erfültt ist, 1 äßt sich der ARIMA $(p, d, q)$-ProzeB als unendicher MA-(AR-)ProzeB formulieren. Der Hauptgrund fur die Kombination der 3 Prozesse in einem Modell liegt im Bestreben, mit möglichst wenigen Parametern möglichst komplizierte Zeitreihen beschreiben zu können (vgl. Möbus und Nagl 1983).

Zur Illustration betrachten wir den unendlichen MA-ProzeB

$$
y_{t}=\frac{{ }_{0}^{\theta}}{1-0,5 B}+a_{t}+a_{t-1}+0,5 a_{t-2}+0,25 a_{t-3}+\ldots
$$

bzw.

$$
Y_{t}=\frac{\theta_{0}}{1-0,5 B}+\frac{(1+0,5 B)}{(1-0,5 B)} \cdot a_{t} .
$$

Der ProzeB G1. (5) ist aber dem $\operatorname{ARIMA}(1,0,1)$-Prozeß mit $\phi_{1}=0,5$ und $\theta_{1}=-0,5$ äquivalent:

$$
(1-0,5 B) y_{t}=\theta_{0}+(1+0,5 B) a_{t} .
$$

Bei der Formulierung eines gemischten Prozesses $(p \neq 0$ und $q \neq 0$ ) sollte man die Gefahr der oberparametrisierung einkalkulieren (Box u. Jenkins 1976, Kap. 7.3.5).

\subsubsection{Modellidentifikation:}

Unter Identifikation von ARIMA-Modellen versteht man die Festlegung der Prozeßparameter $p$, d und $q$ vor der Parameterschätzung. Dabei stiutz sich die Identifikation auf die Autokorrelationsfunktion (PACF). Die ACF ist definiert als:

$$
\rho(k)=\frac{r(k)}{r(0)}, k=0,1,2, \ldots
$$




$$
\text { mit } \gamma(k)=E\left[\left(Y_{t}-\mu_{y}\right)\left(Y_{t+k}-\mu_{y}\right)\right] \text {. }
$$

Die Autokorrelation miBt die Größe des Zusammenhangs der Zeitreihe mit der um $k$ Zeitpunkte verschobenen Zeitreihe. Ein gebräuchlicher Schätzer für die ACF ist:

$$
A C F(k)=r_{k}=\frac{C(k)}{C(0)}, 0 \leq k \leq T-1
$$

mit der Kovarianz

$$
C(k)=\frac{1}{T^{*}} \cdot \sum_{t=1}^{T-k}\left(Y_{t}-\bar{Y}\right)\left(Y_{t+k}-\bar{Y}\right)
$$

$$
\begin{aligned}
& \text { mit } T^{*}=T \\
& \text { bzw. } T^{*}=T-k(\text { Box u. Jenkins 1976) } \\
& \text { McCleary u. Hay 1980). }
\end{aligned}
$$

Mit zunehmend größerem Lag $k$ wird dabei die Schätzung unsicherer, da die Zahl der Kreuzprodukte in G7. (9) mit wachsendem k ständig abnimmt.

Die PACF läßt sich am besten durch eine Analogie zur schrittweisen multiplen Regression erklären, wobei der partielle Autokorrelationskoeffizient jeweils dem letzten Gewicht der Regressionsgleichung entspricht. Unter der Annahme der Stationarität ( $\left(\mathrm{Y}_{t}\right)=\mu_{t}$ für alle t) folgt:

$$
\begin{aligned}
& y_{t}=\phi_{11} y_{t-1}+a_{t} \\
& y_{t}=\phi_{21} y_{t-1}+\phi_{22} y_{t-2}+a_{t} \\
& y_{t}=\phi_{31} y_{t-1}+\phi_{32} y_{t-2}+\phi_{33} y_{t-3}+a_{t} \\
& \cdots \\
& \underline{\text { mit } y_{t}=y_{t}-\mu_{y} .}
\end{aligned}
$$

$\phi_{\text {kk }}$ spiegelt den direkten Einfluß des Zeitpunktes t-k auf $t$ wider. Die Folge $\phi_{11}, \phi_{22}, \phi_{33}, \ldots$ stellt die PACF dar. Die Schätzung der $\phi_{k k}$ erfolgt über die Yule-Walker-Gleichungen (Box u. Jenkins 1976; Nelson 1973): 


$$
\left[\begin{array}{l}
{ }_{k} 1 \\
\phi_{k 2} \\
\vdots \\
\phi_{k k}
\end{array}\right]=\left[\begin{array}{cccc}
1 & r_{1} & r_{2} & \cdots r_{k-1} \\
r_{1} & 1 & r_{1} & \cdots r_{k-2} \\
\vdots & \vdots & \vdots & \ddots \\
r_{k-1} & r_{k-2} & r_{k-3} & \cdots 1
\end{array}\right]-1\left[\begin{array}{c}
r_{1} \\
r_{2} \\
\vdots \\
r_{k}
\end{array}\right] .
$$

Diese Gleichungen besitzen eine Paraliele bei der Bestimmung von Regressionskoeffizienten in der multiplen Regression (s. auch Cooley u. Lohnes 1971, S. 53).

Revenstorf $u$. Keeser (1979, S. 195) geben die in Abb. 1 dargestellte Zusammenfassung für die Identifikation von ARIMA-Modellen.

Zunächst wird die ACF inspiziert, um d festzulegen. In der Literatur (Box U. Jenkins 1976; Glass et al. 1975) wird als Kriterium für Nichtstationarität $i$ Level angegeben, daß die ACF einen besonders flachen Verlauf nimmt. Für die Bestimmung von $p$ und $q$ werden dann die $A C F$ und PACF der d-fach differenzierten Zeitreihe untersucht. Die ACF eines ARIMA(p,d,0)-Prozesses setzt sich u.a. aus einem gedämpften exponentiellen Abfall und/oder gedämpften sinusschwingungen zusammen, während für die theoretische PACF $\phi_{k k} \neq 0$ für $k \leq p$ und $\phi_{k k}=0$ für $k>p g i l t$.

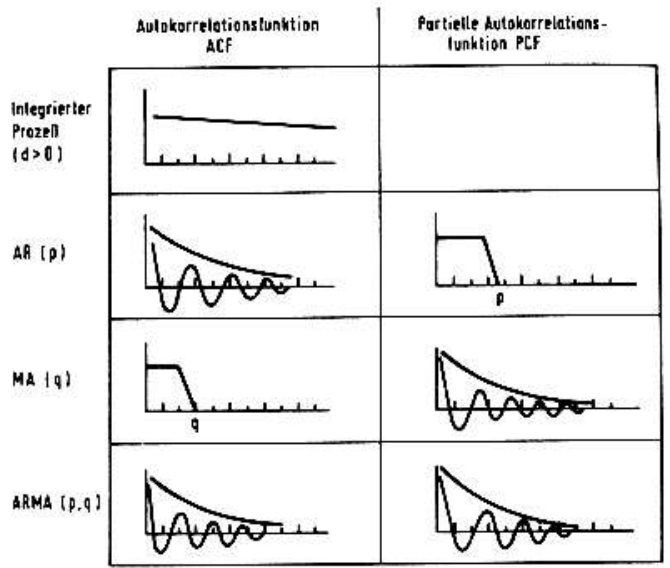

Abb. 1. Grobschema zur Identifikation von ARIMA-Prozessen nach Box u. Jenkins. (Aus Revenstorf u. Keeser 1979, S. 195; Nachdruck mit Genehmigung des Verlags Urban \& Schwarzenberg)

Die PACF eines ARIMA(0,d,q)-Prozesses verhält sich wie die ACF beim $\operatorname{ARIMA}(p, d, 0)-\operatorname{Proze} B$, während für die $\operatorname{ACF} A C F(k) \neq 0$ für $k \leq q$ und $A C F(k)=0$ für $k>q$ gilt.

Bei gemischten Prozessen ( $p \neq 0$ und $q \neq 0$ ) haben sowohl die ACF als auch die PACF eine unendliche Ausdehnung. 
Da die ACF und PACF mit Daten geschätzt werden, versucht man, die empirisch erhaltenen Muster mit den theoretischen zu vergleichen, um so die Grade $p, d, q$ des ARIMA-Modells festzulegen. Daher gehen in die Identifikationsphase stark subjektive Momente ein (Chatfield 1975, S. 25). Einen Hinweis, inwieweit sich die empirische ACF und PACF von den theoretischen Funktionen unterscheiden, geben die Verteilungen der $r_{k}$ und $\phi_{k k}$. Nach Bartlett (1946) gilt:

$$
\vec{\sigma}\left[r_{k}\right]=\frac{1}{\sqrt{T}} \cdot\left\{1+2 \cdot \sum_{i=1}^{q} r_{i}^{2}\right\}^{1 / 2}, k>q \text {, }
$$

sowie nach Quenouilie (1949):

$$
\tilde{\sigma}\left[\phi_{k k}\right]=\frac{1}{\sqrt{\top}} \text { für } k>p \text {. }
$$

Mit GI. (12) und (13) lassen sich Konfidenzintervalle konstruieren. Gilt $\left|r_{k}\right|>2 \cdot \hat{\theta}\left[r_{k}\right]$ bzw. $\left|\phi_{k k}\right\rangle 2 \cdot \hat{0}\left[\hat{\phi}_{k k}\right]$, so sind die $r k$ für $k>q$ bzw. die $\phi_{k k}$ für $k>p$ auf dem 5-\%-Niveau signifikant verschieden von Null.

\subsubsection{Parameterschätzung:}

Der vorläufigen Identifikation schließt sich die Schätzung der Parameter an. Für die Bestimmung der Maximumlikelihood-Schätzer gibt es u. a.:

1. die "Conditional-least-squares-(CLS-)Methode", die auf Aström und Bohtin (1966) zuriuckgeht und von Anderson (1975, 1978) weiter untersucht wurde;

2. die "Unconditional-least-squares-Methode", auch "Backforecasting-Methode" genannt (Box u. Jenkins 1976).

Beide Methoden stellt z.B. das Programmaket BMDP für die Parameterschätzung zur Verfügung. Die geschätzten Parameter müssen innerhalb der Stationaritäs- und Invertferbarkeitsgrenzen liegen. In einem überparametrisierten Modell muissen die uberflüsigen Parameter nicht signifikant sein.

\subsubsection{Modelldiagnostik:}

Nach der Identifikation und der Parameterschätzung wird die Adäquatheit des stochastischen Modells getestet. Fü die "Richtigkeit" eines Modells spricht $v$.a., daß die Residuen at des Modells einem Hhitenoise-ProzeB folgen. Un zu uberprüfen, ob die gesarte ACF nicht signifikant von der ACF eines thite-noise-Prozesses abweicht, wurde von Box und Pierce (1970) der sog. Portemanteau-Test vorgelegt:

$$
Q=(T-d) \cdot \sum_{i=1}^{k} r^{2} \sim x^{2} d f=k-p-q .
$$


Ist Q signifikant, muß die Nullhypothese verworfen werden. Verschiedene Autoren empfehlen, für $k$ den Wert 20-30 zu wählen, da $Q$ sehr sensitiv von $k$ abhängt ( $s$, auch Box u. Jenkins 1976, S. 291).

Weitere Prüfungen des vorläufigen Modells schließen die Berechnung des multiplen Hertes $R^{2}$ als $M a ß$ für die Datenanpassung ein:

$$
R^{2}=1-\sum_{t=1}^{T}\left(\frac{a_{t}^{2}}{y_{t}^{2}}\right)
$$

$$
\begin{aligned}
& \text { mit } y_{t}=\tau_{t}-\frac{\theta_{0}}{1-\phi_{1}-\cdots-\phi_{p}} \\
& (1-B)^{d_{Y_{t}}}-\frac{{ }_{0}}{1-\phi_{1}-\cdots-\phi p}
\end{aligned}
$$

Weitere praktische Hinweise zur Anpassung von ARIMA(p, d, q)-Modellen finden sich be $i$ Glass et al. (1975), Anderson (1975), Gottman $u$. Glass (1978), Makridakis $u$. Wheelwright (1978a, b), Revenstorf und Keeser (1979), Revenstorf (1979), McCain u. McCleary (1979) und Gottman (1981).

\subsubsection{Die Modellierung des Interventionsprozesses}

Während der Ansatz von Box und Tiao (1965) und G1ass et al. (1975) stark am allgemeinen linearen Modell orientiert ist, haben Box und Tiao (1975) die Interventionskomponente konsequent in das Zeitreihenmodell integriert. Einen Vergleich sowie Querverbindungen zwischen den Modellansätzen von Glass et al. (1975) und Box und Tiao (1975) geben Möbus et al. (1983). In dieser Arbeit wird auch gezeigt, daß sich der Modellansatz von Glass et al. (1975) aus dem Box-Tiao-Modell herleiten läßt.

Das Interventionsmodel 1 von Box und Tiao (1975) kennt 3 Variablensätze:

1. die Interventionsvariable I, die zu bestimmten a priori festgelegten Zeitpunkten die Werte 0 oder 1 annimmt, je nachder ob ein Interventionseffekt "ein-" oder "ausgeschaltet" wird;

2. die Effektvariable $Y_{t}^{*}$, die eine Folge dieser Intervention ist, $\gamma_{t}^{*}=f(I t)$, und

3. die beobachtbare Zeitreihe $Y_{t}$, die einerseits durch die Effektvariable $y_{t}^{\#}$ und andererseits durch anderweitige, nichtkontrollierte, Einflüsse $\mathrm{N}_{t}$ geprägt wird:

$$
y_{t}=\left\{\begin{array}{l}
\text { Interventions } \\
\text { effekte }
\end{array}\right\}+\left\{\begin{array}{l}
\text { nichtkontrollierte } \\
\text { Effekte }
\end{array}\right\} .
$$


Die nichtkontrollierten Einflüsse $\mathrm{N}_{t}$, die die Interventionseffekte Überlagern, lassen sich durch ein ARIMA-Modell repräsentieren. Box und Tiao (1975) geben die folgende formale Darstellung von G1. (16):

$$
Y_{t}=\underbrace{\frac{\Omega(B)}{\delta(B)}}_{Y_{t}^{*}} \cdot I_{t}+\underbrace{\frac{\theta_{0}+\theta(B) a_{t}}{(1-B)^{d} \phi(B)}}_{N_{t}} .
$$

Aus 61 . (17) folgt:

$$
\delta(B) Y_{t}^{*}=\Omega(B) I_{t}
$$

bzw. mit $\Omega(B)=\omega(B) B^{b}$

$$
\delta(B) Y_{t}^{*}=\omega(B) I_{t-b}
$$

bzW. mit Hilfe des Backshiftoperators

$$
\left(1-\delta_{1} B-\ldots-\delta_{r} B^{r}\right) Y_{t}^{*}=\left(\omega_{0}-\omega_{1} B-\ldots-\omega_{s} B^{S}\right) I_{t-b}
$$

b Zw.

$$
Y_{t}^{*}=\delta_{1} Y_{t-1}^{*}+\ldots+\delta_{r} Y_{t-r}^{*}+\omega_{0} I_{t-b}-\ldots-\omega_{s} I_{t-b-s} .
$$

Dabei spiegeln die Indizes $r$ und $s$ das "Gedächtnis" der Interventionskomponenten wider. Der Index b gibt an, daB die Intervention sich nit einer Zeitverzögerung von b Zeiteinheiten auswirkt.

Das Transfermodell G1. (18) ist stabil (d.h. explodiert nicht), wenn für die Lösungen der charakteristischen Gleichung

$$
\begin{aligned}
& 1-\delta_{1} \lambda-\ldots-\delta_{r} \lambda^{r}=0 \\
& \left|\lambda_{i}\right|>1 \text { für } i=1,2, \ldots, r \text { gilt. }
\end{aligned}
$$

Die Stabilitätsgrenzen entsprechen numerisch den Grenzen der Stationarität von autoregressiven Prozessen.

In der Literatur unterscheidet man bei der Indikatorvariablen $I_{t}$ zwischen einem Stepinput:

$$
I_{t}=\left\{\begin{array}{l}
0 \text { für } t<t_{1} \\
1 \text { für } t \geq t_{1}
\end{array}\right.
$$




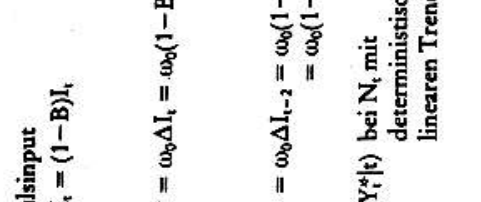

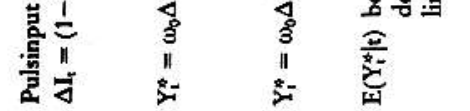

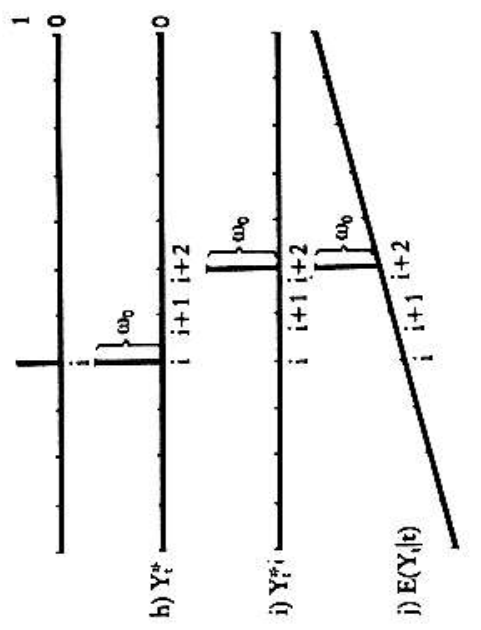

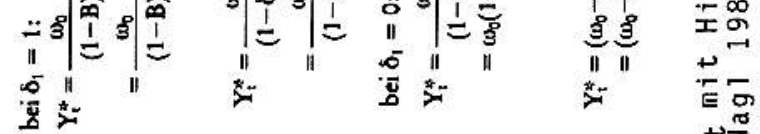

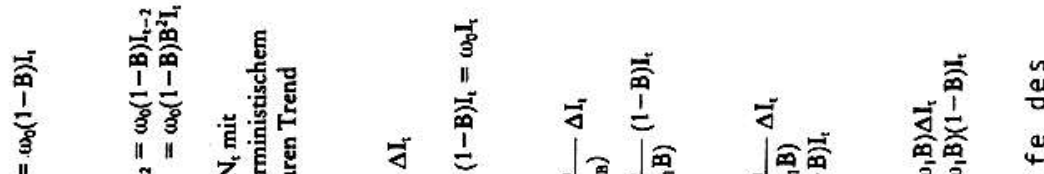

$\stackrel{n}{\circ}$

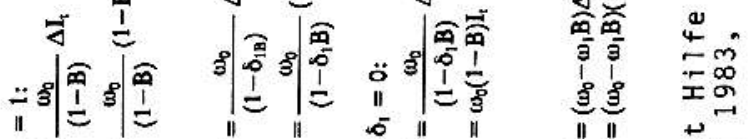

त्र

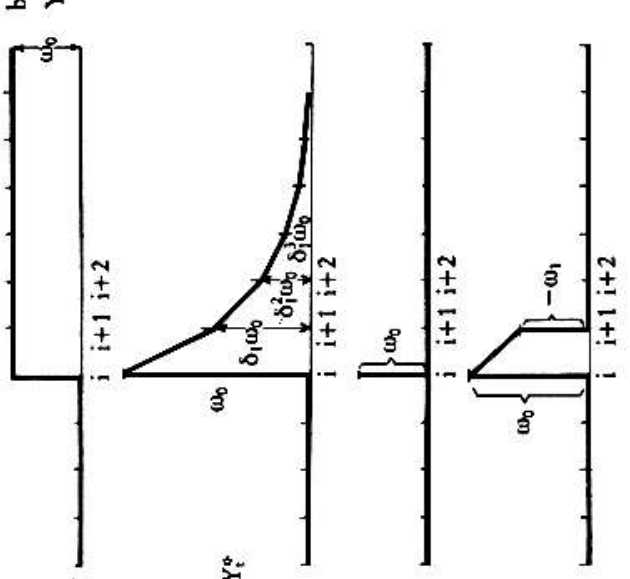

$+\frac{\pi}{2}$

$>$

05

$\stackrel{+}{*}$ o

क्ष

$4: 0$

a

n

능

$\div \leq$

ㄷ.

$\sum \dot{\mathrm{E} 1}$

4

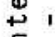

$\leq-1$

$5=\overparen{4}$

d \&

듣 닌

등

站 $=$ ล

-

onn

ร

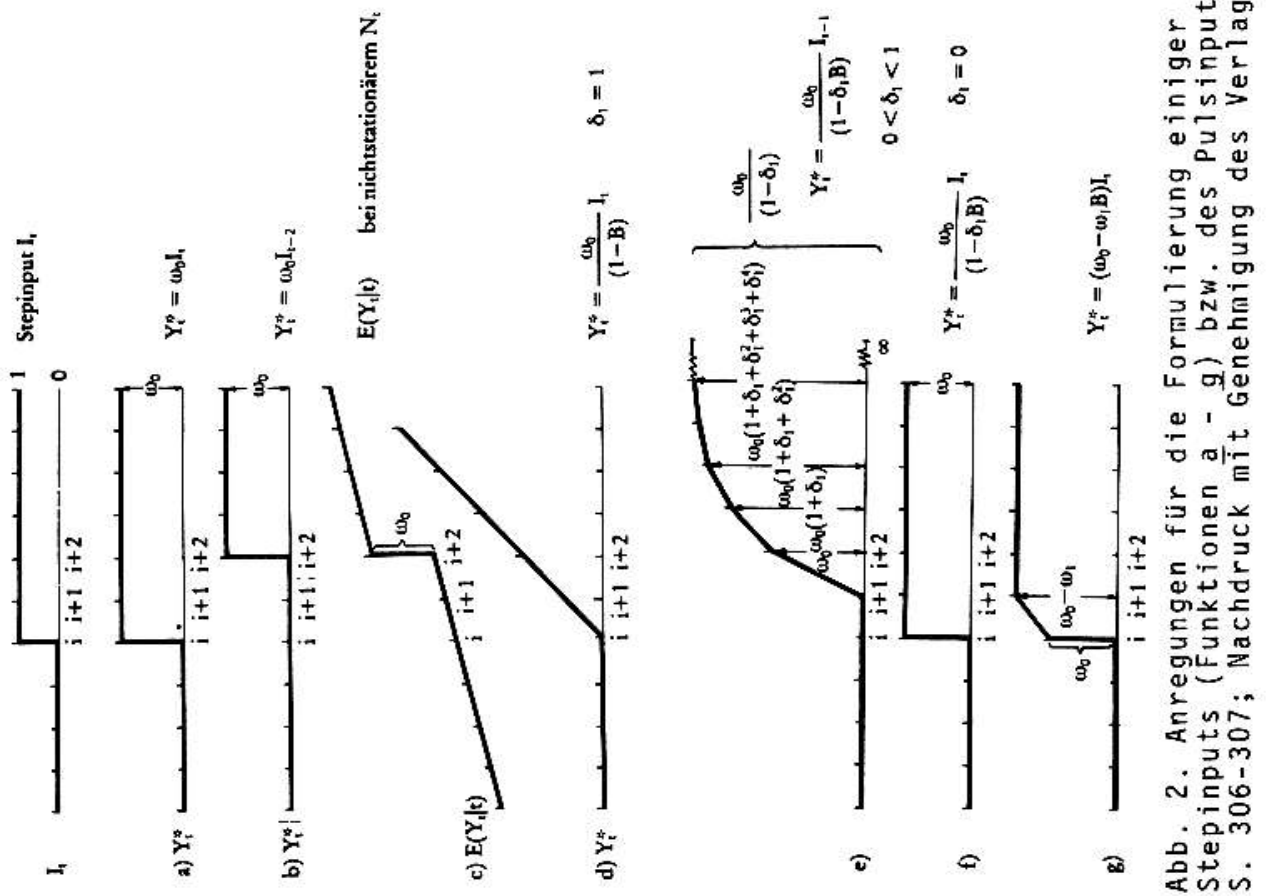


und efnem Pulsinput:

$$
\Delta I_{t}=(1-B)^{d} I_{t}=\left\{\begin{array}{l}
0 \text { für } t \neq t_{1} \\
1 \text { für } t=t_{1}
\end{array} .\right.
$$

In Gi. (20) und (21) bezeichnet $t_{1}$ den ersten MeBzeitpunkt nach Durchführung der Intervention.

Die Abb. 2 zeigt Anregungen für die Postulierung einiger grundlegender Interventionshypothesen. Weitere Erläuterungen hierzu finden sich bei Möbus und $\mathrm{Nagl}(1983, \mathrm{~S}, 305 \mathrm{ff}$.). Die Abb. 3 zeigt eine Abfolge zur Pifung von einfachen Interventionshypothesen.

Zunächst gilt es, ein adäquates ARIMA-Modell fuir den NoiseprozeB zu bestimmen. Die Autoren Box u. Tiao (1975) und Hibbs (1977) schlagen hierzu vor, entweder die gesamte Zeitreihe oder nur die entsprechenden Beobachtungen vor der Intervention zu verwenden.

Nach Cook u. Campbel1 (1979) wird danach das formulierte Interventionsmodell zum identifizierten Noisemodell addiert und alle Parameter des Transfer-ARIMA-Model1s G1. (17) geschätzt. Schi ieß1ich wird dieses Modell analog der Modelldiagnostik in Abschnitt 12.3.1.3 auf Adäquatheit geprüft sowie ein Signifikanztest für die Interventionsparameter durchgeführt.

Liegen $k$ Interventionen vor, so lautet das allgemeine Transfermodell von Box und Tiao (1975):

$$
Y_{t}=\sum_{j=1}^{k} Y_{j t}^{*}+N_{t}=\sum_{j=1}^{k} \frac{\Omega_{j}(B)}{\delta_{j}(B)} I_{j t}+N_{t} .
$$

\section{4 EIN EMPIRISCHES BEISPIEL}

Zu Illustrationszwecken betrachten wir die Zeitreihe für die täglichen Messungen der Hahrnehmungsgeschwindigkeit bei einem schizophrenen Patienten, die aus einer Studie von Meffert stamt (vgl. Holtzman 1963).

Die Zeitreihe in Abb, 4 enthält über den betrachteten Zeitraum von 180 Tagen eine exogene Treatmentvariable mit Langzeitintervallen von 60 Tagen $z$ wischen den Treatmentänderungen.

Der Patient erhielt in den ersten 60 Tagen ein Placebo (Phase I). In den folgenden 60 Tagen wurde das Placebo durch Chlorpromazin ersetzt (Phase II). Vom 120. bis zum 179. Tag (Phase III) wurde die Chlorpromazinbehandlung fortgesetzt, allerdings überlagert von einer Elektroschocktherapie. Da die Phasen I - III "genügend viele" Daten umfassen, wird die Bestimmung eines adäquaten Noisemodells zunächst für jede Phase getrennt durchgefüht, um so zu erkennen, ob die Interventionen eine strukturelle Veränderung des der Zeitreihe zugrunde liegenden ARIMA-Prozesses bewirkt haben. 


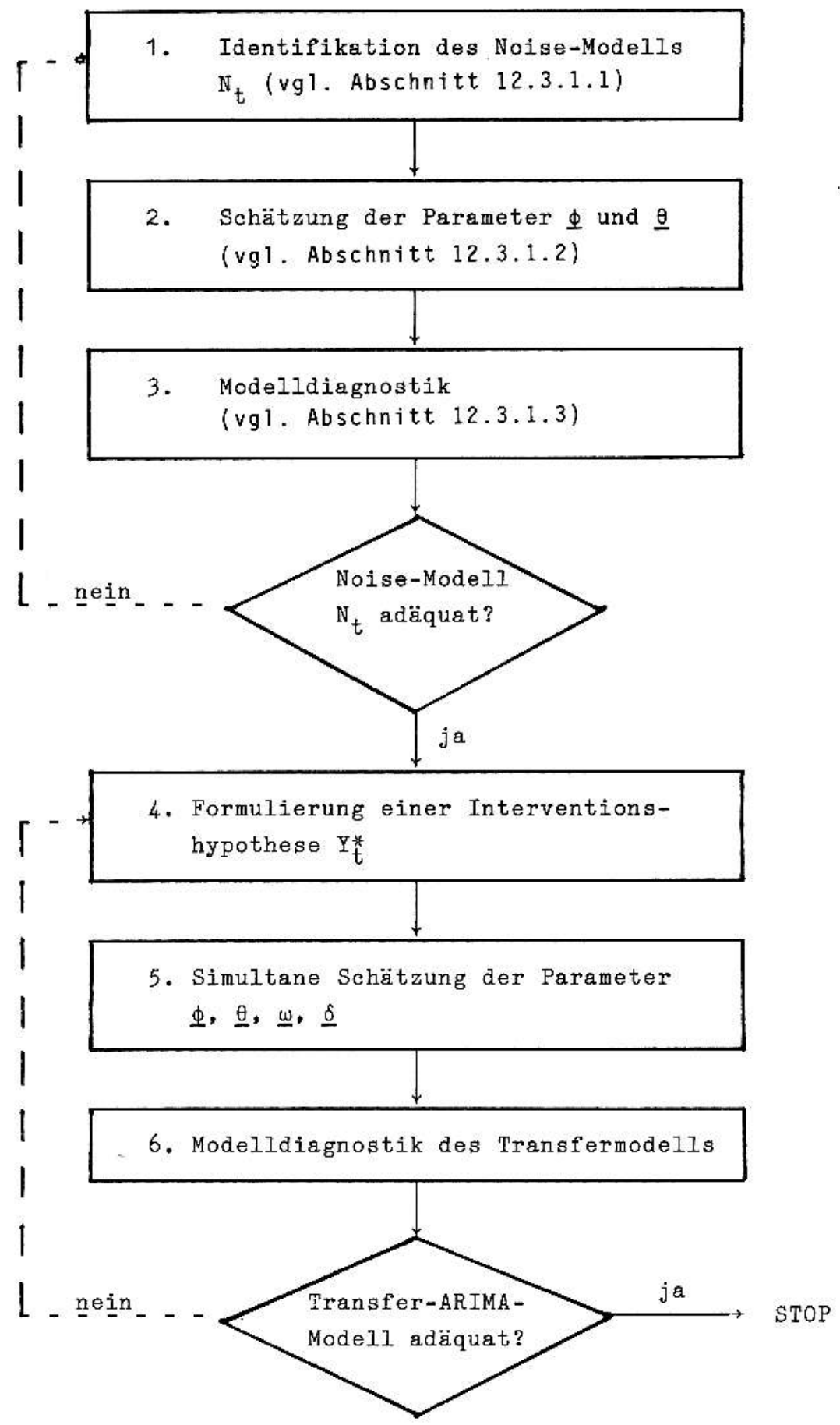

Abb. 3. Schematische Darstellung zur Oberprïfung von Interventionshypothesen nach Box und Tiao 
Die Identifikation, Parameterschätzung und Modelldiagnostik (vgl. Abschn. 12.3.1) ergeben, daß für jede Phase das $\operatorname{ARIMA}(0,1,1)$-Model 1

$$
(1-B) Y_{t}=\theta_{0}+\left(1-\theta_{1} B\right) a_{t}
$$

mit $\theta_{1}=0,76$ und damit für die gesamte Zeitreihe adäquat ist. Diese Homogenitätsprüfung ähnelt dabei der Homogenitätsprüfung in der Kovarianzanalyse oder der Strukturbruchanalyse in der Regression (SchneeweiB 1974). Der Graph der Zeitreihe zeigt deutliche Niveauschwankungen, was ebenfalls auf Nichtstationarität bezüglich des Niveaus ( $d=1)$ schließen läßt. Aus Gl. (23) folgt:

$$
Y_{t}=\frac{\theta_{0}+\left(1-\theta_{1} B\right) a_{t}}{(1-B)}=N_{t} \text {. }
$$

Aus Abb. 4 ist zu erkennen, daß mit der Verabreichung des Tranquilizers ein Levelsprung einsetzt. Für die 2. Intervention wurde zunächst ein einfaches Interventionsmodel1 mit einem Levelsprung am 120 . Tag angenommen. Die geschätzten Residuen wichen signifikant von Whitenoise-Residuen $a b$. Daher werden für die Intervention jetzt 2 Effekte angenommen:

1. eine vorubbergehende Niveauerhöhung vom 120. bis zum 131. Tag und

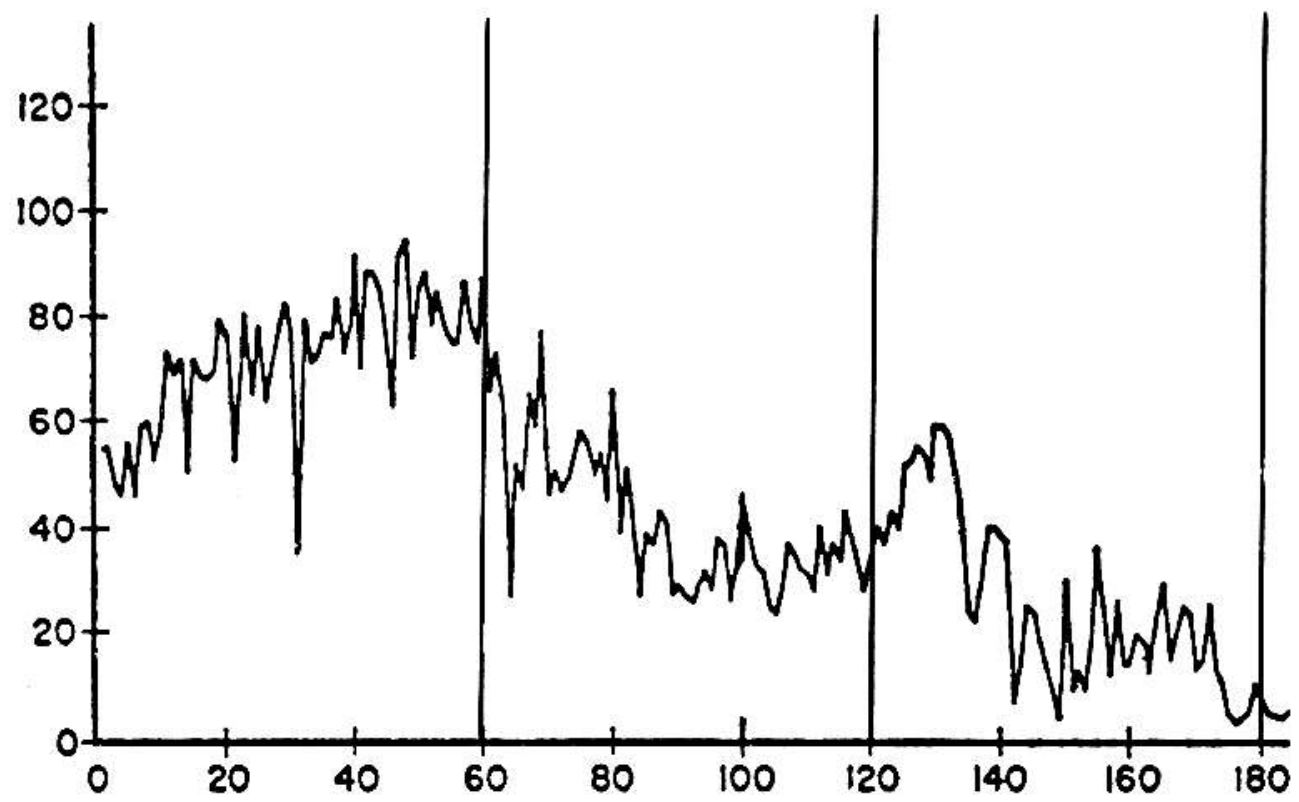

Abb. 4. Tägliche Messungen der Wahrnehmungsgeschwindigkeit bei einem schizophrenen Patienten. (Aus Holtzman 1963) 
2. vom 132. bis zum 180. Beobachtungszeitpunkt ein exponentielles Abfallen der Wahrnehmungsgeschwindigkeit.

Die formale Darstellung der Interventionshypothese lautet:

$$
Y_{t}^{*}=\omega_{0} I_{t}^{(60)}+\omega_{1}\left(I_{t}^{(120)}-I_{t}^{(131)}\right)+\frac{\omega_{2}}{\left(1-\delta_{1} B\right)} B^{12} I_{t}^{(120)} .
$$

Das Interventionsmodell nach G1. (17) ergibt sich zu:

$$
\begin{aligned}
& Y_{t}=Y_{t}^{\star}+N_{t} \\
& =\omega_{0} I_{t}^{(60)}+\omega_{1}\left(I_{t}^{(120)}-I_{t}^{(131)}\right)+\frac{\omega_{2}}{\left(1-\delta_{1} B\right)} B^{12} I_{t}^{(120)} \\
& +\frac{\theta_{0}+\left(1-\theta_{1} B\right) a_{t}}{(1-B)} .
\end{aligned}
$$

Die simultane Schätzung der Parameter des Interventionsmodells G1. (26) liefert $\hat{\theta}_{0}=-2.099 ; \hat{\theta}_{1}=0,76 ; \hat{\omega}_{0}=-21.95 ; \hat{\omega}_{1}=9,09$; $\hat{\omega}_{2}=-4,618$ so wie $\delta_{1}{ }^{\circ}=0,998$. Bis auf $\theta_{0}$ sind alle Parameter signifikant verschieden von Null.

Im folgenden wollen wir die Auswertung des Zeitreihenexperiments von Meffert mit Hilfe des Randomisierungstests demonstrieren. Nach der Beobachtungsphase (Basislinie) A erfolgt die 1. Behandlungsphase B1, die direkt von der 2. Intervention $B 2$ abgelöst wird. Somit liegt dem Experiment ein $A B_{1} B_{2}$-Design zugrunde.

Um beim Randomisierungstest die approximative Prüfverteilung anwenden zu können, müssen jedoch mindestens 4 A- und 4 B-Phasen vorliegen (vgl. Möbus u. Nagl 1983, S. 292). Da jede Phase des A B 1 B2-Designs 60 Daten umfaBt, wird daher jede dieser Phasen in 4 Teilphasen zu jeweils 15 Beobachtungswerten eingeteilt, so daB wir ein

$$
\begin{aligned}
& A_{1} A_{2} A_{3} A_{4}\left|\quad B_{1} B_{2} B_{3} B_{4}\right| \quad B_{5}{ }^{B} 6^{B} 7^{B} 8^{-D e s i g n ~ e r h a l t e n . ~} \\
& I_{1} \quad I_{2}
\end{aligned}
$$

Die Zusammenfassung von jeweils 15 Tagen zu einer Einheit wird hier nicht inhaltlich begründet, sondern ist aus dem Wunsch zu erklären, prinzipiell die Nullhypothese verwerfen zu können. Dies wäre bei nur 3 Phasen nicht mehr möglich. Die Null-und die gerichtete Alternativhypothese lauten:

$H_{0}$ : Kein Unterschied zwischen A- und B-Phase,

$$
\begin{aligned}
& \mathrm{H}_{1}: \overline{\mathrm{Y}}_{\mathrm{B}_{8}}=\overline{\mathrm{Y}}_{\mathrm{B}_{7}}=\overline{\mathrm{Y}}_{\mathrm{B}_{6}}=\overline{\mathrm{Y}}_{\mathrm{B}_{5}}<\overline{\mathrm{Y}}_{\mathrm{B}_{4}}=\overline{\mathrm{Y}}_{\mathrm{B}_{3}}=\overline{\mathrm{Y}}_{\mathrm{B}_{2}}=\overline{\mathrm{Y}}_{\mathrm{B}_{1}} \\
&<\overline{\mathrm{Y}}_{\mathrm{A}_{4}}=\overline{\mathrm{Y}}_{\mathrm{A}_{3}}=\overline{\mathrm{Y}}_{\mathrm{A}_{2}}=\overline{\mathrm{Y}}_{\mathrm{A}_{1}} .
\end{aligned}
$$


Dabei gilt im einzelnen:

$$
\begin{array}{llll}
\bar{Y}_{B_{8}}=13,4 & \bar{Y}_{B_{7}}=19,33 & \bar{Y}_{B_{6}}=23,933 & \bar{Y}_{B_{5}}=50,0, \\
\bar{Y}_{B_{4}}=34,067 & \bar{Y}_{B_{3}}=31,4 & \bar{Y}_{B_{2}}=42,867 & \bar{Y}_{B_{1}}=55,6, \\
\bar{Y}_{A_{4}}=80,53 & \bar{Y}_{A_{3}}=76,267 & \bar{Y}_{A_{2}}=71,533 & \bar{Y}_{A_{1}}=58,26 .
\end{array}
$$

Aufgrund der Struktur von $\mathrm{H}_{1}$ erhalten die künstlichen Phasenmittelwerte in einem ordinalen Gewichtungsschema die Gewichte:

$$
\begin{aligned}
& g_{B_{i}}=1 \text { für } i=1,2,3,4, \\
& g_{B_{j}}=2 \text { für } j=5,6,7,8, \\
& g_{A_{k}}=3 \text { für } k=1,2,3,4 .
\end{aligned}
$$

Gemäß GI. (2) erhalten wir:

$$
z_{\text {emp }}=\frac{\Gamma_{\text {emp }}-E(\Gamma)}{\sqrt{\operatorname{Var}(\Gamma)}}=\frac{1244,331-1114,344}{64,064}=2,029 .
$$

Bei einseitiger Fragestellung auf dem 5-\%-Niveau ist dieses Ergebnis signifikant.

\section{LITERATUR}

Anderson $0 D$ (1975) Time series analysis and forecasting: The Box-Jenkins approach. Butterworths, London

Anderson TW (1978) Repeated measurements in autoregressive processes. J An Statist AssoC $73: 371-378$

Aström KJ, Bohlin T (1966) Numerical identification of linear dynamic systems from normal operating records. In: hammond $\mathrm{PH}$ (ed) Theory of self-adaptive control systeris. Plenum, New York

Bartlett MS (1946) On the theoretical specifications of the sampling properties of autocorrelated time series. J R Statist Soc B $8: 27-41$

Box GEP, Jenkins GH (1976) Time series analysis: Forecasting and control. Holden-Day, San Francisco 
BoX GEP, Pierce DA (1970) Distribution of residual autocorrelations in ARIMA tine series models. J Am Statist Assoc 64:1509-1526

Box GEP, Tiao GC (1965) A change in level of a non-stationary timeseries. Biometrika 52:181-192

Box GEP, Tiao GC (1975) Intervention analysis with applications to economic and environmental problems. J Am Statist Assoc 70:70-79

Chatfield C (1975) The analysis of time-series: Theory and practice. Chapman \& Hall, London

Cook TD, Campbell DT (1979) Quasi-experiments: Nonequivalent control groups designs. In: Cook TD, Campbel1 TD (eds) Quasi-experimentation: Design and analysis issues for field settings. McNally, Chicago, pp 95-146

Cooley WW, Lohnes PR (1971) Multivariate data analysis. Wiley \& Sons, New York

Edgington ES (1967) Statistical inference from $N=1$ experiments. J Psychol 65:195-199

Edgington ES (1969a) Statistical inference: The distribution free approach. McGraw-Hill, New York

Edgington ES (1969b) Approximate randomization tests. J Psychol 72:143-149

Edgington ES (1971) Randomization tests with statistical control over conconitant variables. J Psychol 79:13-19

Edgington ES (1973) Randomization tests: Computer time requirements. J Psychol 85:89-95

Edgington ES (1975a) Randomization tests for one-subject operant experiments. J Psychol 90:57-68

Edgington ES (1975b) Randomization for predicted trends. Can Psychol Rev 16:49-53

Edgington ES (1980) Randomization tests. Dekker, New York

Fisher RA (1951) The design of experiments. Hafner, London

Glass GV, Hilison UL, Gottman JM (1975) Design and analysis of timeseries experiments. Colorado Associated University Press, Boulder

Gottman JH (1981) Time-series analysis. Cambridge University Press, Cambridge

Gottman JM, Glass GV (1978) Analysis of interrupted time-series experiments. In: Kratochwill TR (ed) Single subject research: Strategies for evaluating change. Academic Press, New York, p p $197-235$

Hibbs DA (1977) On analyzing the effects of policy interventions: BOX-JENKINS and BOX-TIAO vs. structural equation models. In: Heise DR (ed) Sociological methodology. Jossey-Bass, New York, pp $137-179$ 
Holtzman WH (1963) Statistical models for the study of change in the single case. In : Harris $C$ (ed) Problems in measuring change. University of Wisconsin Press, Madison Hisconsin

Jenkins GM (1979) Practical experiences with modelling and forecasting time series. GJP Ltd., Channel Island

Lehman EL (1975) Nonparametrics: Statistical methods based on ranks. Holden-Day, San Francisco

Levin JR,. Marascuilo LA, Hubert LJ (1978) $N=1$ - Nonparametric randomization tests. In: Kratochwill TR (ed) Single subject research. Academic Press, New York, pp 167-196

Makridakis S, Wheelwright SC (1978a) Forecasting - methods and applications. Wiley \& Sons, New York

Makridakis S, Wheelwright SC (1978b) Interactive forecasting. Holden-Day, San Francisco

McCain LJ, McCleary $R$ (1979) The statistical analysis of the simple interrupted time-series quasi-experiment. In: Cook TD, CampbeTT DT (eds) Quasi-experimentation: Design and analysis for field settings. McNally, Chicago, pp 233-293

McCleary R, Hay RA (.1980) Applied time-series analysis for the social sciences. Sage, Beverly Hills

Möbus C, Nagl W (1983) Messung, Analyse und Prognose von Veränderungen. In: Bredenkamp J, Feger $H$ (Hrsg) Hypothesenprüfung, Bd 5 der Serie Forschungsmethoden der Psychologie der Enzyklopädie der Psychologie. Verlag für Psychologie, Göttingen, S 239-470

Möbus C, Göricke G, Kröh PA (1983) Statistical analysis of single-case experimental designs: Conditional equivalence of the general-linear-model approach of Glass, Willson and Gottman with the intervention model of Box and Tiao. EDV in Med Biol $14: 98-108$

Nelson CR (1973) Applied time-series analysis. Holden-Day, San Francisco

Quenouille MH (1949) Approximate tests of correlation in time series. J R Statist Soc B 11:68-74

Revenstorf $D$ (1979) Zeitreihenanalyse für klinische Daten: Methodik und Anwendungen. Beltz, Weinheim

Revenstorf $D$, Keeser $W$ (1979) Zeitreihenanalyse von Therapieverläufen. In: Petermann F, Hehl FJ (Hrsg) Einzelfallanalyse. Urban \& Schwarzenberg, München, S 183-228

SchneeweiB H (1974) Okonometrie, 2. Auf1. Physica, Wïrzburg Wien 